\title{
Stability of Covariant Relativistic Quantum Theory
}

\author{
V. Wessels, W. N. Polyzou*
}

August 6, 2021

\begin{abstract}
In this paper we study the relativistic quantum mechanical interpretation of the solution of the inhomogeneous Euclidean Bethe-Salpeter equation. Our goal is to determine conditions on the input to the Euclidean Bethe-Salpeter equation so the solution can be used to construct a model Hilbert space and a dynamical unitary representation of the Poincaré group. We prove three theorems that relate the stability of this construction to properties of the kernel and driving term of the Bethe-Salpeter equation. The most interesting result is that the positivity of the Hilbert space norm in the non-interacting theory is not stable with respect to Euclidean covariant perturbations defined by Bethe-Salpeter kernels. The long-term goal of this work is to understand which model Euclidean Green functions preserve the underlying relativistic quantum theory of the original field theory. Understanding the constraints imposed on the Green functions by the existence of an underlying relativistic quantum theory is an important consideration for formulating field-theory motivated relativistic quantum models.
\end{abstract}

\section{Introduction}

The purpose of this paper is to investigate the conditions for field-theory motivated calculations based on covariant Euclidean Green functions to be interpreted as relativistic quantum mechanical theories. The problem is to use the Euclidean Green functions to construct a model Hilbert space and the dynamical unitary representation of the Poincaré group on this space [1]. There are two long-term goals of this research. The first goal is to understand which field-theory motivated calculations can be interpreted as relativistic quantum

${ }^{*}$ This work supported in part by the U.S. Department of Energy, under contract DE-FG0286ER40286 
theories. The second goal is to learn how to formulate phenomenological relativistic Euclidean quantum models which have a clear connection to an underlying quantum field theory.

This paper focuses on Euclidean rather than Minkowski Green functions for two reasons. First, Euclidean Green functions are used extensively in applications, including lattice discretizations of quantum field theories 2], perturbative quantum field theory, and in the Schwinger-Dyson equations [3]. The second reason for considering Euclidean Green functions is that they are directly related to the underlying relativistic quantum theory.

The relation of Euclidean Green functions to relativistic quantum theory is discussed in the literature on axiomatic field theory. A readable summary of this relationship can be found in section 1.3 of 2 . More complete treatments appear in the original literature [4, [5], 6]. Reference [4] contains a clear statement of assumptions needed to reconstruct a relativistic quantum theory from a collection of Euclidean Green functions. Reference [5] contains a concise alternative treatment in terms of Euclidean generating functionals.

This paper investigates the quantum mechanical interpretation of the inhomogeneous Euclidean Bethe-Salpeter equation. This is the simplest model where the relation to an underlying relativistic quantum theory can be addressed. In addition, it is an important tool for making field-theory motivated models of two-body systems. The input to the Bethe-Salpeter equation consists of the Bethe-Salpeter kernel and the two-point Euclidean Green function. In applications this input is unknown and has to be modeled. A reasonable goal is to find conditions on the model input that are sufficient to construct a relativistic quantum mechanical two-body model. Even this modest goal turns out to be a non-trivial problem. In this paper we prove three theorems that address the relation of the phenomenological input to the existence of an underlying relativistic quantum theory.

The first two theorems establish an interesting result, which is that if the driving term of the inhomogeneous Bethe-Salpeter equation for the Green functions is constructed from the free Euclidean Green functions, and the four-point Green function is constructed by solving the Bethe-Salpeter equation with a small Euclidean covariant kernel, the positivity of the resulting Hilbert space norm is unstable with respect to small variations in the kernel. This was contrary to our expectation that a sufficiently small Euclidean covariant kernel would always preserve the underlying quantum mechanical interpretation of the non-interacting system. The third theorem shows that the instability proof breaks down if the free two-point function is replaced by a more realistic twopoint function that has a Källén-Lehmann weight with a continuous component to its mass spectrum.

\section{Background}

The Euclidean formulation of quantum field theory plays a central role in this paper. This section contains a summary of the relationship between Euclidean 
quantum field theory, relativistic quantum mechanics, and Minkowski Green functions. While all of this material can be found in the literature, the focus in this section is on aspects of these relationships which are needed to formulate relativistic quantum mechanical models which are not local field theories.

There are three classes of generalized functions that are important in this paper. The first class are the Wightman functions, which are vacuum expectation values of products of local field operators:

$$
W_{n}\left(x_{1}, \cdots, x_{n}\right):=\left\langle 0\left|\phi_{1}\left(x_{1}\right) \cdots \phi_{n}\left(x_{n}\right)\right| 0\right\rangle .
$$

The second class of generalized functions are the Minkowski Green functions, which are time-ordered vacuum expectation values of products of local field operators

$$
G_{n}\left(x_{1}, \cdots, x_{n}\right):=\left\langle 0\left|T\left(\phi_{1}\left(x_{1}\right) \cdots \phi_{n}\left(x_{n}\right)\right)\right| 0\right\rangle .
$$

The third class of generalized functions are the Schwinger functions, or $\mathrm{Eu}-$ clidean Green functions, which will be defined later as analytic continuations of the Minkowski Green functions or Wightman functions.

The transformation properties of the Wightman functions and Minkowski Green functions are needed to construct unitary representations of the Poincaré group. The Wightman and Minkowski Green functions are Poincaré covariant. For the Wightman function this is a consequence of the transformation properties of the fields and the invariance of the vacuum. For the Minkowski Green functions locality is also needed to ensure the Poincaré invariance of the time-ordered product of fields.

The symmetry group for the fields is inhomogeneous $S L(2, C)$. The group $S L(2, C)$ is the covering group for the Lorentz group. The $S L(2, C)$ matrices $\pm \Lambda$ are related to the Lorentz transformation $\Lambda^{\mu}{ }_{\nu}$ by

$$
\Lambda_{\nu}^{\mu}=\frac{1}{2} \operatorname{Tr}\left(\Lambda \sigma_{\mu}\left(\Lambda^{*}\right)^{t} \sigma_{\nu}\right),
$$

where $\sigma_{\mu}=(I, \vec{\sigma})$ and $\vec{\sigma}$ are the Pauli matrices.

Covariant fields are multicomponent operator densities that transform as finite dimensional representations, $D\left(\Lambda, \Lambda^{*}\right)$, of $S L(2, C)$ :

$$
U(\Lambda, a) \phi(x) U^{\dagger}(\Lambda, a)=\phi(\Lambda x+a) D\left(\Lambda, \Lambda^{*}\right) .
$$

where $\Lambda$ is an element of $S L(2, C)$ and $\Lambda x+a$ is a short-hand notation for $\Lambda^{\mu}{ }_{\nu} x^{\nu}+a^{\mu}$. In $S L(2, C)$ the $\Lambda$ and $\Lambda^{*}$ define inequivalent representations and both are needed to construct the irreducible representations of the Lorentz group and the corresponding representations of the four-dimensional orthogonal group.

The resulting covariance properties of the Wightman functions and Minkowski Green functions are

$$
W_{n}\left(x_{1}, \cdots, x_{n}\right)=W_{n}\left(\Lambda x_{1}+a, \cdots, \Lambda x_{n}+a\right) \prod_{i=1}^{n} D_{i}\left(\Lambda, \Lambda^{*}\right)
$$


and

$$
G_{n}\left(x_{1}, \cdots, x_{n}\right)=G_{n}\left(\Lambda x_{1}+a, \cdots, \Lambda x_{n}+a\right) \prod_{i=1}^{n} D_{i}\left(\Lambda, \Lambda^{*}\right),
$$

respectively.

The Hilbert space and dynamical unitary representation of the Poincaré group [1] of the field theory is determined by the Wightman functions. This is illustrated with the following example of a vector obtained by applying the product of smeared field operators to the physical vacuum:

$$
|\Psi\rangle:=\phi(f) \phi(g)|0\rangle \quad \phi(f):=\int d^{4} x \phi(x) f(x)
$$

where there is an implied sum over the components of $f$ and $\phi$. The Hilbert space scalar product of $|\Psi\rangle$ and $|\Psi\rangle^{\prime}$ is related to a four-point Wightman function by

$$
\begin{gathered}
\left\langle\Psi \mid \Psi^{\prime}\right\rangle:= \\
\int d x d x^{\prime} d y d y^{\prime} g^{*}\left(x^{\prime}\right) f^{*}\left(y^{\prime}\right) W\left(x^{\prime}, y^{\prime} ; y, x\right) f^{\prime}(y) g^{\prime}(x)
\end{gathered}
$$

where

$$
W\left(x^{\prime}, y^{\prime} ; y, x\right):=\left\langle 0\left|\phi_{2}^{\dagger}\left(x^{\prime}\right) \phi_{1}^{\dagger}\left(y^{\prime}\right) \phi_{1}(y) \phi_{2}(x)\right| 0\right\rangle .
$$

It is normally assumed that polynomials in the fields evaluated on Schwartz test functions applied to the vacuum are a dense set of vectors in the Hilbert space. Schwartz functions [ 7 8 are infinitely differentiable functions which decrease faster than any inverse polynomial. Continuous multilinear functionals on products of Schwartz functions are tempered distributions. All of the generalized functions in this paper are assumed to be tempered distributions. This is a mild assumption which ensures the analytic properties that are traditionally assumed in quantum field theories.

The scalar product of vectors constructed by applying more complex polynomials of smeared fields applied to the vacuum can also be expressed in terms of Wightman functions.

The transformation properties of the state (7) follow from the covariance (4) of the fields

$$
U(\Lambda, a)|\Psi\rangle=\phi_{1}\left(D_{1}\left(\Lambda, \Lambda^{*}\right) f^{\prime}\right) \phi_{2}\left(D_{2}\left(\Lambda, \Lambda^{*}\right) g^{\prime}\right)|0\rangle
$$

where $f^{\prime}(x)=f\left(\Lambda^{-1}(x-a)\right)$ and $g^{\prime}(x)=g\left(\Lambda^{-1}(x-a)\right)$.

The Poincaré invariance of the Hilbert space scalar product

$$
\left\langle U(\Lambda, a) \Psi \mid U(\Lambda, a) \Psi^{\prime}\right\rangle=\left\langle\Psi \mid \Psi^{\prime}\right\rangle
$$

follows if the test functions have the transformation property

$$
U(\Lambda, a) f(x)=D\left(\Lambda, \Lambda^{*}\right) f\left(\Lambda^{-1}(x-a)\right) .
$$


This illustrates how the covariance of the Wightman functions leads to a unitary representation of the Poincaré group on the Hilbert Space defined by the Wightman functions.

The structure of the Hilbert space scalar product in (8) is not limited to relativistic quantum field theory; it is the general form of the Hilbert-space scalar product in any relativistic quantum theory where the Poincaré group is implemented by manifestly covariant [9] transformations of test functions like $f$ and $g$. This suggests the possibility of formulating "approximations" to the underlying field theory that remain relativistic quantum theories.

The construction of the relativistic quantum dynamics defined by generalizations of (18), (10) and (12) can be compared to the familiar textbook construction of Fock space Poincaré generators based on Noether's theorem. The Noether's theorem construction is limited to free fields. The introduction of an interaction leads to "generators" that are not densely defined operators on the Fock space. They do not have self-adjoint extensions and attempts to regularize these "generators" so they become self-adjoint operators invariably lead to violations of the commutation relations. The Wightman-function construction agrees with the textbook construction for free fields, and it is not limited to perturbation theory or Lagrangian field theory for interacting systems.

While the Wightman functions determine the quantum interpretation of the field theory, they are difficult to use in models because they do not have inverses. This is apparent from the structure of the Fourier transform of the two-point Wightman function of a scalar field theory, which is, up to a constant, the product of a mass-shell delta function and a positive energy Heaviside function. In spite of their role in defining the underlying quantum theory, Wightman functions have not been extensively used in practical applications.

The Minkowski Green functions have the advantage that as Green functions, they are expected to have inverses, which make it possible to derive useful equations, like the Bethe-Salpeter equation. The disadvantage of these Green functions is that they are not directly related to the underlying quantum theory. Their connection to a quantum theory is that for each time-ordering the Minkowski Green function agrees with the Wightman function whose fields have the same order as the Minkowski times. The quantum theory can be recovered if there is enough analyticity to reconstruct the Wightman functions from the limited information contained in the Minkowski Green function.

In quantum field theories the Euclidean Green functions or Schwinger functions are related to the Wightman functions and Minkowski Green functions by analytic continuation. Schwinger functions are important because (1) they exist under mild conditions (2) they can be used to directly construct the underlying relativistic quantum theory and (3) they are expected to have inverses. The original use of analytic continuation of the Fourier transforms of the Minkowski Green functions to imaginary energies is due to Dyson [10, who used the analytic continuation as a tool to study ultraviolet divergences in perturbation theory.

In 1958 Schwinger [11 used the spectral condition of the physical intermediate states and Poincaré covariance to show that the $n$-point Minkowski Green 
functions of a quantum field theory can be analytically continued to imaginary times:

$$
S_{n}\left(\mathrm{x}_{1}, \cdots, \mathrm{x}_{n}\right):=\lim _{\phi: 0 \rightarrow \pi / 2}=G_{n}\left(\vec{x}_{1}, e^{-i \phi} t_{1}, \cdots, \vec{x}_{n}, e^{-i \phi} t_{n}\right) .
$$

In equation (13) and the remainder of this paper $x=\left(x^{0}, \vec{x}\right)$ denotes a real Minkowski four vector and $x=\left(x^{0}, \vec{x}\right)$ denotes a real Euclidean four vector. The components of these vectors are related by

$$
\mathrm{x}_{k}:=\left(\mathrm{x}^{0}, \vec{x}_{k}\right)=\left(-i x_{k}^{0}, \vec{x}_{k}\right) \quad x_{k}:=\left(x^{0}, \vec{x}_{k}\right)=\left(i \mathrm{x}_{k}^{0}, \vec{x}_{k}\right) .
$$

This analytic continuation (13) defines the Schwinger functions for noncoincident times, $t_{i} \neq t_{j}$. The existence of this analytic continuation is based on a multi-variable generalization [12] of the Payley-Wiener-type theorem that states that

$$
\tilde{f}\left(t-t^{\prime}\right):=\int d E f(E) e^{-i E\left(t-t^{\prime}\right)}
$$

can be analytically continued to the lower-half $\left(t-t^{\prime}\right)$-plane if $f(E)$ is a tempered distribution with support for positive energies. In the definition (13) the order of the Euclidean times is the same as the order of the Minkowski times in the Minkowski Green functions. The covariance properties of the Schwinger functions $S_{n}$ are not apparent from the definition (13) and will be discussed separately.

The Fourier representation of $G_{n}\left(x_{1}, \cdots, x_{n}\right)$ can be used to extend the analytic continuation in (13) to complex $\left(x_{i}-x_{i+1}\right)$ with $-\left(x_{i}-x_{i+1}\right) \in R^{4}+$ $i \mathcal{V}^{+}$, where $\mathcal{V}^{+}$is the open future-pointing light cone. On this domain the real part of $i p_{i} \cdot\left(x_{i}-x_{i+1}\right)$ is negative when $p_{i}$ is time-like with positive energy, which ensures the analyticity. This domain is called the tube in the literature 13. In principle each time ordering of the Minkowski Green function has a different analytic continuation. The different analytic continuations are defined on disjoint domains characterized by different Euclidean time orderings. Also, because for each time ordering the Minkowski Green function is equal to the Wightman function with the fields ordered in the same order as the times, each analytic continuation is the analytic continuation of a different Wightman function.

Since the Wightman functions transform covariantly with respect to a finite dimensional representation of the Lorentz group, (5), the complexification of the covariance condition can be used to extend the domain of analyticity from the tube to the domain generated from the tube by complex Lorentz transformations, called the extended tube 13 .

This extension is done using the complex Lorentz group. Complex Lorentz transformations are complex linear transformations that leave the Minkowski line element invariant. In the appendix it is shown that the most general complex Lorentz transformation connected to the identity has the representation

$$
\Lambda(A, B)^{\mu}{ }_{\nu}=\frac{1}{2} \operatorname{Tr}\left(A \sigma_{\mu} B^{t} \sigma_{\nu}\right)=\Lambda_{\nu}^{\mu}(A, B)
$$


where $A$ and $B$ are independent $S L(2, C)$ transformations. The covering group of the complex Lorentz groups is $S L(2, C) \times S L(2, C)$. Real Lorentz transformations are obtained by taking $A=B^{*}=\Lambda$. The connection with Euclidean covariance follows because four-dimensional complex orthogonal transformations have a representation similar to (16),

$$
E^{\mu}{ }_{\nu}(A, B)=\frac{1}{2} \operatorname{Tr}\left(A \tilde{\sigma}_{\mu} B^{t} \tilde{\sigma}_{\nu}^{\dagger}\right)
$$

where $\tilde{\sigma}_{\mu}=\left(i \sigma_{0}, \vec{\sigma}\right)$. In the Euclidean case real $O(4)$ transformations have $A, B \in S U(2)$. It follows from (16) and (17) that the covering group of the complex orthogonal group in four dimensions is also $S L(2, C) \times S L(2, C)$. Complex covariance can be used to extend the analytic continuation to all points $x_{i}^{\prime}=\Lambda(A, B)^{-1}\left(x_{i}-a\right)$ that can be reached from points in the tube by complex Lorentz transformations using

$$
\begin{gathered}
W_{n}\left(x_{1}^{\prime}, \cdots, x_{n}^{\prime}\right)= \\
W_{n}\left(x_{1}, \cdots, x_{n}\right) \prod_{i=1}^{n} D_{i}(\Lambda(A, B))
\end{gathered}
$$

which is consistent with real Lorentz covariance when $A=\Lambda=B^{*}$ and with real Euclidean covariance when $A, B \in S U(2)$. Restricting to real Euclidean points in the extended tube and letting $A, B \in S U(2)$ leads to real Euclidean invariance of each of the $n$ ! analytic continuations.

In quantum field theory the $n$ ! analytic continuations are defined on common domains consisting of open sets of real Minkowski space-like separated points called Jost points [13. If the Wightman functions are constructed from local fields the analytic continuations have to be identical on the Jost points because the order of the fields is irrelevant (up to sign for fermions) on space-like separated points. The result is a that all of the $n$ ! analytic continuations are part of a single-valued Euclidean covariant Green function with transformation properties

$$
\begin{gathered}
S_{n}\left(\mathrm{x}_{1}^{\prime}, \cdots, \mathrm{x}_{n}^{\prime}\right)= \\
S_{n}\left(\mathrm{x}_{1}, \cdots, \mathrm{x}_{n}\right) \prod_{i=1}^{n} D_{i}(\Lambda(A, B))
\end{gathered}
$$

with $\mathrm{x}^{\mu \prime}=E(A, B)^{\mu}{ }_{\nu} \mathrm{x}^{\nu}+\mathrm{a}^{\mu}$. This defines what we mean by Euclidean covariance.

The real Euclidean transformation properties of the Schwinger functions are obtained from those of the corresponding Wightman functions by replacing the pair $\left(\Lambda, \Lambda^{*}\right)$ by the $S U(2)$ matrices $(A, B)$ :

$$
D\left(\Lambda, \Lambda^{*}\right) \rightarrow D(A, B)
$$

An important property of the Schwinger functions is that they can be used to directly formulate the underlying quantum theory. This was first done in 
1973 by Nelson [14, who also related the Schwinger functions to moments of a Euclidean functional integral. A more useful construction was given during the same year by Osterwalder and Schrader 4 6]. Fröhlich 5 gave an elegant reformulation of the Osterwalder-Schrader construction in terms of Euclidean generating functionals. In 4] Osterwalder and Schrader identified properties of Schwinger functions that are sufficient to reconstruct the underlying relativistic quantum field theory. They exploited the relation between the Schwinger functions and the Wightman functions, which are the boundary values of the analytic continuation:

$$
W_{n}\left(x_{1} \cdots x_{n}\right)=\lim _{\mathrm{x}_{1}^{0}>\cdots>\mathrm{x}_{n}^{0} \rightarrow 0} S_{n}\left(\overrightarrow{\mathrm{x}}_{1}, \mathrm{x}_{1}^{0}-i x_{1}^{0}, \cdots, \overrightarrow{\mathrm{x}}_{n}, \mathrm{x}_{n}^{0}-i x_{n}^{0}\right) .
$$

There are $n$ ! Wightman functions depending on the ordering of the $n$ fields; the ordering on the Euclidean times in the limit (21) selects the Wightman function with the fields ordered in the same order as the Euclidean times. In equation (21) there are no restrictions on the Minkowski times in the resulting Wightman function. The other $n !-1$ Wightman functions are selected by taking the limit of the Schwinger function with different orderings on the Euclidean times. The $n$ ! Wightman functions agree with $n$ ! analytic functions generated by the $n$ ! time orderings of the Minkowski Green functions.

By exploiting this relationship Osterwalder and Schrader were able to find conditions on the Schwinger functions that are, up to some technical growth conditions [6], equivalent to the axioms given by Wightman. Furthermore, Osterwalder and Schrader were able to identify an independent subset of axioms 44 that are sufficient to construct the Hilbert space and a unitary representation of the Poincaré group with four momentum satisfying a spectral condition.

The relevant conditions on the Schwinger functions are that they should be Euclidean covariant (19) tempered distributions which satisfy a property called reflection positivity. Reflection positivity will be discussed more completely in the next section. It is used to construct the physical Hilbert space scalar product and is most simply illustrated with an example that is analogous to the example (8). In the Osterwalder Schrader approach the scalar product (8) is replaced by

$$
\begin{gathered}
\left\langle\Psi \mid \Psi^{\prime}\right\rangle= \\
\int d \mathrm{x} d \mathrm{x}^{\prime} d \mathrm{y} d \mathrm{y}^{\prime} g^{*}\left(\theta \mathrm{x}^{\prime}\right) f^{*}\left(\theta \mathrm{y}^{\prime}\right) S\left(\mathrm{x}^{\prime}, \mathrm{y}^{\prime} ; \mathrm{y}, \mathrm{x}\right) f^{\prime}(\mathrm{y}) g^{\prime}(\mathrm{x})
\end{gathered}
$$

where $f(\mathrm{x}), g(\mathrm{x})$ are functions of four Euclidean space-time variables with disjoint positive-Euclidean-time support for $0<\mathrm{y}^{0}<a<x^{0}<b<\infty$ and $\theta \mathrm{x}=\left(-\mathrm{x}^{0}, \overrightarrow{\mathrm{x}}\right)$ is the Euclidean time-reversal operator. Here $a$ and $b$ are constants that serve to separate the Euclidean time support of $f$ and $g$. This sesquilinear form is the physical Hilbert space scalar product in the Osterwalder Schrader formalism. In field theories the ordering on the support of the Euclidean times is equivalent to choosing an order of the fields in the Wightman functions. Reflection positivity requires that (22) is non-negative when $|\Psi\rangle=\left|\Psi^{\prime}\right\rangle$. Like the Wightman case there are generalizations of (22) to more complicated states. 
The surprising feature of (22) is the physical Hilbert space can be directly defined in terms of the Schwinger functions, with no need to transform back to the Minkowski formulation of the Hilbert space based on Wightman functions.

In the next section we discuss how the Euclidean covariance of reflection positive Schwinger functions can be used to construct a unitary representation of the Poincaré group on the Hilbert space defined by the scalar product (22).

This paper is concerned with a relativistic quantum theory which is not necessarily a local quantum field theory. In applications where locality is relaxed, assumptions need to be made about the relationship between the Wightman functions, the Minkowski Green functions, and the Schwinger functions. In the absence of locality the $n$ ! Wightman functions are no longer required to be related. The Minkowski Green function does not necessarily have a single valued analytic continuation. To proceed it is useful to assign a fundamental significance to one of the three classes of generalized functions and use this to formulate a quantum theory.

In this paper we always assume that the model Schwinger functions have a more fundamental status. The model Schwinger functions are taken to be single valued tempered distributions which satisfy the covariance condition (19). This requirement is motivated by the observation that most of the models of interest lead to single-valued Euclidean covariant Schwinger functions. For example, models generated from Euclidean generating functionals and approximations to Euclidean path integrals naturally lead to single valued Euclidean covariant model Schwinger functions.

Since the model Schwinger functions will not have all of the properties of the Schwinger functions of a local field theory, the relation to model Minkowski Green functions and model Wightman functions cannot be expected to be identical to the relationship found in local field theories. The most important requirement is that the model Schwinger functions will also be assumed to have enough reflection positivity to build a relativistic quantum theory with the desired particle content. The precise formulation of this condition will be discussed in sections three and four.

The focus of this paper is properties of two- and four-point Euclidean Green functions. Reflection positivity imposes conditions on the two and four-point function for them to be part of a system of Schwinger functions that define the Hilbert-space scalar product of a relativistic quantum theory.

The Bethe-Salpeter equation can be understood by considering the cluster property of the four-point Schwinger function, which has the form

$$
S=S_{0}+S_{t}
$$

where $S_{0}$ is a sum of products of two-point Schwinger functions and $S_{t}$ is the truncated four-point Schwinger function. If $S$ and $S_{0}$ can also be understood as Green functions in the traditional sense, i.e. as kernels of integral operators with inverses, then it is possible to define the Bethe-Salpeter Kernel:

$$
K=S_{0}^{-1} S_{t} S^{-1} .
$$


When $K \neq 0$ the four-point Schwinger function $S$ is generated by solving the inhomogeneous Euclidean Bethe-Salpeter equation

$$
S=S_{0}+S_{0} K S
$$

given a Bethe-Salpeter kernel $K$.

In quantum field theory $S, K$, and $S_{0}$ are only known formally and the Bethe-Salpeter equation is a constraint that relates these three quantities. In order to make this into a solvable equation two modifications are normally

made. First, $S_{0}$ is replaced by the $S_{0}$ of a free field theory. The virtue of this "approximation" is that the resulting $S_{0}$ is known. Second, the Bethe-Salpeter kernel $K$ is modeled, using either perturbative methods or theoretically and/or experimentally motivated phenomenological methods. The cluster properties (23) suggest that the kernel should be a short-ranged operator.

In this paper we examine the following stability question. Let $K$ be a sufficiently small, Euclidean covariant, model Bethe-Salpeter kernel and let $S_{0}$ be the Schwinger function of an underlying relativistic quantum theory. Is the solution, $S$, of the Euclidean Bethe-Salpeter equation (25) the $S$ of a relativistic quantum theory? In the absence of such a stability, arbitrarily small uncertainties in the model Bethe-Salpeter Kernel could lead to a theory that is no longer a relativistic quantum theory. In this paper we show that for the special case that $S_{0}$ is the Schwinger function of a free field theory that $S$ can fail to satisfy the constraints imposed by reflection positivity for arbitrarily small Euclidean-covariant kernels $K$. While this result does not apply to the exact Bethe-Salpeter equation, where $S_{0}$ is not the free $S_{0}$, many applications of the Bethe-Salpeter equation "approximate" $S_{0}$ by the free $S_{0}$.

In the next section we summarize the structure of a Euclidean Relativistic quantum theory. We define reflection positivity and review how it is used to construct the physical Hilbert space of the theory and the relativistic quantum dynamics. We identify necessary conditions for reflection positivity that we use to study four-point Schwinger functions in section five. The construction of a free-particle dynamics is illustrated in section four. In section five we show that the necessary conditions for reflection positivity, which are derived in section three, can be violated for arbitrarily small Bethe-Salpeter kernels when $S_{0}$ is the $S_{0}$ of a free-field theory. We also prove a result that suggests that the instability may not appear in the exact Bethe-Salpeter equation. The implications of this result are discussed in section six.

\section{Euclidean Relativistic Quantum Mechanics}

In this section we give a short description of Euclidean relativistic quantum theory. We review how a collection of Euclidean-invariant reflection-positive Schwinger functions are used to construct the physical Hilbert space and the dynamical unitary representation of the Poincaré group of a relativistic quantum theory. A readable description of the main elements of this construction can can be found in 2]. Mathematical treatments of this construction can be found 
in [4] 6] [5]. Self-adjointness of the boost generators can be established using the methods discussed in [16] 17].

For the purpose of illustration we consider the case of Schwinger functions for a scalar field. We discuss the spin $1 / 2$ case in section three.

A relativistic quantum theory is defined by a unitary representation of the Poincaré group [1] acting on the physical Hilbert space with four-momentum generators that have a spectrum in the future-pointing light cone.

As discussed in section two the physical Hilbert space of Euclidean relativistic quantum mechanics is defined by constructing an inner product on a nice set of vectors; limits are used to complete the Hilbert space. As in the Wightman case, the Euclidean Green functions are assumed to yield finite results when integrated against Schwartz test functions [7] of $4 N$ Euclidean space-time variables.

Let $\mathcal{S}$ be the space of finite sequences of Schwartz test functions in different numbers of Euclidean space-time variables:

$$
\langle\mathrm{x} \mid f\rangle:=\left\{f_{0}, f_{1}\left(\mathrm{x}_{11}\right), f_{2}\left(\mathrm{x}_{21}, \mathrm{x}_{22}\right), \cdots, f_{k}\left(\mathrm{x}_{k 1}, \cdots, \mathrm{x}_{k k}\right)\right\}
$$

where $f_{l}\left(\mathrm{x}_{l 1}, \cdots, \mathrm{x}_{l l}\right)$ is a Schwartz function in $l$ Euclidean space-time variables. These functions are the Euclidean replacements for the functions $f$ and $g$ that appear in the Minkowski scalar product (8).

To construct the physical Hilbert space Osterwalder and Schrader 4 introduce the subspace $\mathcal{S}_{>}$of $\mathcal{S}$, where each of the functions $f_{l}$ has support for an ordered set of positive Euclidean times, $x_{l l}^{0}>\cdots>x_{l 1}^{0}>0$. The projection on $\mathcal{S}_{>}$is denoted by $\Pi_{>}$. The space $\mathcal{S}_{>}$is natural for two reasons. First, each Euclidean time-ordering defines a scalar product that is equal to the corresponding Minkowski scalar product defined in terms of the Wightman function with the fields ordered in the same order as the Euclidean times. Second, the ordering has a well-defined Minkowski limit if the Wightman functions are consistent with requirements imposed by the spectral condition.

The Euclidean time-reversal operator $\Theta$ on $\mathcal{S}$ is defined by

$$
\langle x \mid \Theta f\rangle:=\left\{f_{0}, f_{1}\left(\theta \mathrm{x}_{11}\right), f_{2}\left(\theta \mathrm{x}_{21}, \theta \mathrm{x}_{22}\right), \cdots, f_{k}\left(\theta \mathrm{x}_{k 1}, \cdots, \theta \mathrm{x}_{k k}\right)\right\} .
$$

where $\theta\left(x^{0}, \vec{x}\right):=\left(-x^{0}, \vec{x}\right)$.

Given a collection of Euclidean-covariant Schwinger functions

$$
\left\{S_{n}\left(\mathrm{x}_{1}, \cdots, \mathrm{x}_{n}\right)\right\},
$$

which are tempered distributions, and test functions $f, g \in \mathcal{S}_{>}$, Osterwalder and Schrader define the quadratic form

$$
\begin{gathered}
(\Theta f, S g)=(f, \Theta S g):= \\
\sum_{m, n} \int d^{4} \mathrm{x}_{1} \cdots d^{4} \mathrm{x}_{m+n} f_{m}^{*}\left(\theta \mathrm{x}_{m}, \cdots, \theta \mathrm{x}_{1}\right) S_{m+n}\left(\mathrm{x}_{1}, \cdots, \mathrm{x}_{m+n}\right) g_{n}\left(\mathrm{x}_{m+1}, \cdots, \mathrm{x}_{m+n}\right) .
\end{gathered}
$$


The support conditions on the functions $f$ and $g$ select the part of the Schwinger function that has a given Wightman function as the boundary value of an analytic function.

The relation $f \sim g$ if and only if

$$
(\Theta(f-g), S(f-g))=0 .
$$

defines an equivalence relation on $\mathcal{S}_{>}$. The functions $f \in \mathcal{S}_{>}$can be put into disjoint equivalence classes with respect to this equivalence relation; the equivalence class containing $f \in \mathcal{S}_{>}$is denoted by $[f]_{\sim}$. The equivalence class containing zero is denoted by $[0]_{\sim}$. The equivalence relation is $S$ dependent.

The sesquilinear form (29) is well-defined on equivalence classes:

$$
\left\langle[f]_{\sim} \mid[g]_{\sim}\right\rangle=(\Theta f, S g)
$$

where $f$ and $g$ are any representatives of $[f]_{\sim}$ and $[g]_{\sim}$ respectively.

Reflection positivity is the condition that

$$
\left\|[f]_{\sim}\right\|^{2}:=\left\langle[f]_{\sim} \mid[f]_{\sim}\right\rangle=(\Theta f, S f) \geq 0
$$

and vanishes only for $[f]_{\sim}=[0]_{\sim}$.

A dense set of vectors in the physical Hilbert space is the space of equivalence classes of functions $[f]_{\sim} \in \mathcal{S}_{>}$. The physical Hilbert-space inner product of two vectors is given by (31), where the inner product can be evaluated using any $f \in[f]_{\sim}$ and $g \in[g]_{\sim}$. The physical Hilbert space is obtained by completing the space of equivalence classes in the norm defined by (32).

This defines the physical Hilbert space directly in terms of the Schwinger functions. Reflection positivity is equivalent to the statement that vectors in the physical Hilbert space have positive length.

The involution, $\Theta$, on the Euclidean space serves as a "conjugation operator". We will show how this "conjugation" converts a representation of a subgroup of the complex Euclidean group into a unitary representation of the Poincaré group.

A necessary condition for reflection positivity is that it holds on subspaces of $\mathcal{S}_{>}$. This ensures that vectors restricted to subspaces also have positive length. The subspaces of most relevance to the Bethe-Salpeter equation are subspaces generated by equivalence classes containing positive-time functions of one or two Euclidean space-time variables. Reflection positivity implies the following constraints on the two- and four-point Schwinger functions

$$
\begin{gathered}
\int d^{4} \mathrm{x}_{1} d^{4} \mathrm{x}_{2} f_{1}^{*}\left(\theta \mathrm{x}_{1}\right) S_{2}\left(\mathrm{x}_{1}, \mathrm{x}_{2}\right) f_{1}\left(\mathrm{x}_{2}\right) \geq 0 . \\
\int d^{4} \mathrm{x}_{1} \cdots d^{4} \mathrm{x}_{4} f_{2}^{*}\left(\theta \mathrm{x}_{2}, \theta \mathrm{x}_{1}\right) S_{4}\left(\mathrm{x}_{1}, \mathrm{x}_{2}, \mathrm{x}_{3}, \mathrm{x}_{4}\right) f_{2}\left(\mathrm{x}_{3}, \mathrm{x}_{4}\right) \geq 0
\end{gathered}
$$

The relevant observation is that the two- and four-point Schwinger functions must define a positive scalar product on the subspaces defined above. We test these conditions in section four. 
To complete the construction of a relativistic quantum theory we need to construct a unitary representation of the Poincaré group on the physical Hilbert space.

The Poincare group is a subgroup of the complex Poincaré group, which also contains the real orthogonal group in four space-time dimensions. The infinitesimal generators of Euclidean transformations and Poincaré transformations are related by complex multiplication. Euclidean time-translations and rotations in Euclidean space-time planes correspond to Poincaré time translations with imaginary times, and rotationless Lorentz boosts with imaginary rapidity, respectively. While these finite transformations are unitary with respect to a Euclidean scalar product, they are Hermitian with respect to the physical scalar product (31). This is the reason that the $\Theta$ appears in the definition of the physical scalar product. The identity of the complex Lorentz group and complex $O(4)$ is discussed in the appendix.

The infinitesimal forms of spatial translation, rotations, Euclidean time translations, and Euclidean space-time rotations are used to identify the infinitesimal generators of the Poincaré group. The operators $H, \vec{P}, \vec{J}$ and $\vec{B}$ satisfy the commutation relations of the Poincaré Lie algebra:

$$
\begin{gathered}
\langle x|H| f\rangle:= \\
\left\{0, \frac{\partial}{\partial \mathrm{x}_{11}^{0}} f_{1}\left(\mathrm{x}_{11}\right),\left(\frac{\partial}{\partial \mathrm{x}_{21}^{0}}+\frac{\partial}{\partial \mathrm{x}_{22}^{0}}\right) f_{2}\left(\mathrm{x}_{21}, \mathrm{x}_{22}\right), \cdots\right\} \\
\langle\mathrm{x}|\vec{P}| f\rangle:= \\
\left\{0,-i \frac{\partial}{\partial \overrightarrow{\mathrm{x}_{11}}} f_{1}\left(\mathrm{x}_{11}\right),-i\left(\frac{\partial}{\partial \overrightarrow{\mathrm{x}_{21}}}+\frac{\partial}{\partial \overrightarrow{\mathrm{x}_{22}}}\right) f_{2}\left(\mathrm{x}_{21}, \mathrm{x}_{22}\right), \cdots\right\} \\
\langle\mathrm{x}|\vec{J}| f\rangle:= \\
\left\{0,-i \overrightarrow{\mathrm{x}}_{11} \times \frac{\partial}{\partial \overrightarrow{\mathrm{x}}_{11}} f_{1}\left(\mathrm{x}_{11}\right),-i\left(\overrightarrow{\mathrm{x}}_{21} \times \frac{\partial}{\partial \overrightarrow{\mathrm{x}}_{21}}+\overrightarrow{\mathrm{x}}_{22} \times \frac{\partial}{\partial \overrightarrow{\mathrm{x}}_{22}}\right) f_{2}\left(\mathrm{x}_{21}, \mathrm{x}_{22}\right), \cdots\right\} \\
\langle\mathrm{x}|\vec{B}| f\rangle:= \\
\left\{0,\left(\overrightarrow{\mathrm{x}}_{11} \frac{\partial}{\partial \mathrm{x}_{11}^{0}}-\mathrm{x}_{11}^{0} \frac{\partial}{\partial \overrightarrow{\mathrm{x}}_{11}}\right) f_{1}\left(\mathrm{x}_{11}\right),\right. \\
\left.\left(\overrightarrow{\mathrm{x}}_{21} \frac{\partial}{\partial \mathrm{x}_{21}^{0}}-\mathrm{x}_{21}^{0} \frac{\partial}{\partial \overrightarrow{\mathrm{x}}_{21}}+\overrightarrow{\mathrm{x}}_{22} \frac{\partial}{\partial \mathrm{x}_{22}^{0}}-\mathrm{x}_{22}^{0} \frac{\partial}{\partial \overrightarrow{\mathrm{x}}_{22}}\right) f_{2}\left(\mathrm{x}_{21}, \mathrm{x}_{22}\right), \cdots\right\} .
\end{gathered}
$$

Elementary computations show that these operators are Hermitian with respect to the physical scalar product (31). The Poincaré commutation relations imply that these operators are the Hamiltonian, linear momentum operators, the angular momentum operators, and the rotationless Lorentz-boost generators. For both $H$ and $\vec{B}$ the Hermiticity follows because $\Theta$ changes the sign of the Euclidean time.

When the model-Schwinger functions are covariant, rather than invariant, and the discrete "field" indices transform with respect to a finite-dimensional 
irreducible representation $D(A, B)$ of $S L(2, C) \times S L(2, C)$ then the expression for the rotation and Lorentz boost generators need to be modified

$$
\begin{aligned}
\left(-i \overrightarrow{\mathrm{x}}_{11} \times \frac{\partial}{\partial \overrightarrow{\mathrm{x}}_{11}}\right) & \rightarrow\left(-i \overrightarrow{\mathrm{x}}_{11} \times \frac{\partial}{\partial \overrightarrow{\mathrm{x}}_{11}}+\vec{\Sigma}\right) \\
\left(\overrightarrow{\mathrm{x}}_{11} \frac{\partial}{\partial \mathrm{x}_{11}^{0}}-\mathrm{x}_{11}^{0} \frac{\partial}{\partial \overrightarrow{\mathrm{x}}_{11}}\right) & \rightarrow\left(\overrightarrow{\mathrm{x}}_{11} \frac{\partial}{\partial \mathrm{x}_{11}^{0}}-\mathrm{x}_{11}^{0} \frac{\partial}{\partial \overrightarrow{\mathrm{x}}_{11}}+\overrightarrow{\mathcal{B}}\right)
\end{aligned}
$$

where

$$
\vec{\Sigma}=i \vec{\nabla}_{\phi} D\left(e^{\frac{-i}{2} \vec{\sigma} \cdot \vec{\phi}}, e^{\frac{i}{2} \vec{\sigma}^{t} \cdot \vec{\phi}}\right)_{a a^{\prime}}
$$

and

$$
\overrightarrow{\mathcal{B}}=\vec{\nabla}_{\rho} D\left(e^{\frac{-i}{2} \vec{\sigma} \cdot \vec{\rho}}, e^{\frac{-i}{2} \vec{\sigma}^{t} \cdot \vec{\rho}}\right)_{a a^{\prime}}
$$

where the derivatives are evaluated at $\phi=\rho=0$ and $\vec{\sigma}^{t}$ is the transpose of $\vec{\sigma}$.

We have glossed over two technical points. First, the Euclidean time translations and Euclidean space-time rotations that were used to construct $H$ and $\vec{B}$ do not map $\mathcal{S}_{>}$to $\mathcal{S}_{>}$. For the time translations this is addressed by considering only future pointing Euclidean time translations, which do map $\mathcal{S}_{>}$to $\mathcal{S}_{>}$. Reflection positivity can be used to show that this defines a contractive Hermitian semigroup. Stone's theorem for contractive Hermitian semigroups [19] guarantees that $H$ is a self-adjoint operator on the physical Hilbert space. The contractive nature of the semigroup also implies that the Hamiltonian satisfies the spectral condition. For the Lorentz transformations this is addressed by restricting the domain of the transformations to successively larger positivetime convex cones, $\frac{\Delta \mathrm{x}^{0}}{\Delta|\bar{x}|}<\tan (\phi)$, in $\mathcal{S}_{>}$that map into $\mathcal{S}_{>}$for rotations through sufficiently small angles, $\phi^{\prime}, \phi+\phi^{\prime}<\pi / 2$. Self-adjointness of the generators on the physical Hilbert space is established by showing that these restricted transformations are symmetric local semigroups [16] 17] [18, which necessarily have self-adjoint generators.

The second technicality is that it is necessary to establish that the formal operators are well defined on the equivalence classes that define Hilbert space vectors. This is done by working on suitable domains of functions and integrating by parts. Specifically if $f \in[0]_{\sim}$ and $X: \mathcal{S}_{>} \rightarrow \mathcal{S}_{>}$:

$$
([g], \Theta S[X f])=(g, \Theta S X f)=(X g, \Theta S f)=([X g], \Theta S[f])=0
$$

which shows $f \in[0]_{\sim}$ implies $X f \in[0]_{\sim}$, where $X$ can be any of the operators, $\vec{P}, \vec{J}, \vec{B}$ or $H$.

The result of this construction is a physical Hilbert space and a set of ten selfadjoint operators that satisfy the Poincaré commutation relations. This shows how a collection of Euclidean-covariant, reflection-positive model Schwinger functions can be used to directly define a relativistic quantum dynamics satisfying the spectral condition. Note that all of the computations were performed using only Euclidean space-time variables; the corresponding model Wightman functions are not needed.

Particle exchange symmetry and microscopic locality put additional constraints on model Schwinger functions, but these additional constraints do not impact our stability analysis. 


\section{One-Particle Systems:}

Since this Euclidean formulation of the relativistic quantum theory is abstract and possibly unfamiliar, in this section we show how it leads to familiar results for the case of a free particle of mass $m$.

The two-point Schwinger function for a free field of mass $m$ is

$$
S_{2}(\mathrm{x}-\mathrm{y}):=\frac{1}{(2 \pi)^{4}} \int \frac{d^{4} \mathrm{p}}{\mathrm{p}^{2}+m^{2}} e^{i \mathrm{p} \cdot(\mathrm{x}-\mathrm{y})}
$$

We first demonstrate that $S_{2}(\mathrm{x}-\mathrm{y})$ is reflection positive [20. Let $f(\mathrm{x})$ be any positive-time function of one Euclidean space-time variable. Reflection positivity requires (32)

$$
\left(f, \Theta S_{2} f\right):=\int d^{4} \mathrm{x} d^{4} \mathrm{y} f(\mathrm{x}) S_{2}(\theta \mathrm{x}-\mathrm{y}) f(\mathrm{y}) \geq 0
$$

for all $f(\mathrm{x})$ with positive Euclidean time support.

To demonstrate the inequality (45) note

$$
\begin{gathered}
\left(f, \Theta S_{2} f\right) \\
=\frac{1}{(2 \pi)^{4}} \int d^{4} \times d^{4} \mathrm{y} d^{4} \mathrm{p} f(\mathrm{x}) \frac{e^{i \mathrm{p} \cdot(\theta \mathrm{x}-\mathrm{y})}}{\mathrm{p}^{2}+m^{2}} f(\mathrm{y}) \\
=\frac{1}{(2 \pi)^{4}} \int d^{4} \mathrm{x} d^{4} \mathrm{y} d^{4} \mathrm{p} f(\mathrm{x}) \frac{e^{-i \mathrm{p}_{0} \cdot\left(\mathrm{x}_{0}+\mathrm{y}_{0}\right)+i \overrightarrow{\mathrm{p}} \cdot(\overrightarrow{\mathrm{x}}-\overrightarrow{\mathrm{y}})}}{\left(\mathrm{p}^{0}+i \omega_{m}(\overrightarrow{\mathrm{p}})\right)\left(\mathrm{p}^{0}-i \omega_{m}(\overrightarrow{\mathrm{p}})\right)} f(\mathrm{y})
\end{gathered}
$$

where

$$
\omega_{m}(\overrightarrow{\mathrm{p}}):=\sqrt{m^{2}+\overrightarrow{\mathrm{p}} \cdot \overrightarrow{\mathrm{p}}} .
$$

The $p_{0}$ integral is computed using the residue theorem. The positivity of the Euclidean times means that the contour in the $p_{0}$ integral should be closed in the lower half $\mathrm{p}_{0}$-plane. The result of the contour integral is

$$
\frac{1}{2} \int d^{3} \mathrm{p} \frac{|g(\overrightarrow{\mathrm{p}})|^{2}}{\omega_{m}(\overrightarrow{\mathrm{p}})} \geq 0
$$

where

$$
g(\overrightarrow{\mathrm{p}}):=\frac{1}{(2 \pi)^{3 / 2}} \int d^{4} y f(\mathrm{y}) e^{-\omega_{m}(\overrightarrow{\mathrm{p}}) y_{0}-i \overrightarrow{\mathrm{p}} \cdot \overrightarrow{\mathrm{y}}} .
$$

This is the standard Lorentz invariant scalar product for a particle of mass $m$, with momentum space wave function $g(\overrightarrow{\mathrm{p}})$.

The expressions for the Poincaré generators in (35138) act on the functions $f$, or more properly equivalences classes $[f]_{\sim}$. Using these covariant forms of the generators in equation (49) leads to equivalent forms of the generators as operators acting on the wave functions $g(\overrightarrow{\mathrm{p}})$ :

$$
H=\omega_{m}(\overrightarrow{\mathrm{p}})
$$




$$
\begin{gathered}
\vec{P}=\overrightarrow{\mathrm{p}} . \\
\vec{J}=i \vec{\nabla}_{\mathrm{p}} \times \overrightarrow{\mathrm{p}} \\
\vec{B}=i \omega_{m} \vec{\nabla}_{\mathrm{p}} .
\end{gathered}
$$

These are the familiar forms of the single particle Poincare generators in the representation with the scalar product [48).

Similar results can be obtained for the case of spin $1 / 2$ particles. For spin $1 / 2$ particles the Euclidean two-point Green function is

$$
S_{2}(\mathrm{x}-\mathrm{y}):=\frac{1}{(2 \pi)^{4}} \int d^{4} \mathrm{p} \frac{m-p \cdot \gamma_{e}}{\mathrm{p}^{2}+m^{2}} e^{i \mathrm{p} \cdot(\mathrm{x}-\mathrm{y})}
$$

where

$$
i \gamma_{0 e}=\gamma^{0}=-\gamma_{0} ; \quad \gamma_{e}^{i}=\gamma^{i} .
$$

In this case, because the Minkowski Green function is normally defined with a Dirac conjugate field rather than a Hilbert space adjoint, the $\gamma^{0}$ needs to be eliminated from $S_{2}$ to get the continuation to the Wightman function that serves as the kernel of the Hilbert space scalar product. This can be achieved by introducing $\gamma^{0}$ as the spinor part of the $\Theta$ operator:

$$
\begin{gathered}
\left(f, \Theta \gamma^{0} S_{2} f\right) \\
=\frac{1}{(2 \pi)^{4}} \int d^{4} \mathrm{x} d^{4} \mathrm{y} d^{4} \mathrm{p} f(\mathrm{x}) e^{i \mathrm{p} \cdot(\Theta \mathrm{x}-\mathrm{y})} \gamma^{0} \frac{m-p \cdot \gamma_{e}}{\mathrm{p}^{2}+m^{2}} f(\mathrm{y}) \\
=\int d^{3} \mathrm{p} g^{\dagger}(\overrightarrow{\mathrm{p}}) \frac{\Lambda_{+}(p)}{(2 \pi)^{3}} g(\overrightarrow{\mathrm{p}})
\end{gathered}
$$

where

$$
\Lambda_{+}(p):=\frac{\omega_{m}(\vec{p})+\gamma^{0} \vec{\gamma} \cdot \vec{p}-m \gamma^{0}}{2 \omega_{m}(\vec{p})}
$$

is the positive energy Dirac projector and

$$
g(\vec{p}):=\int d^{4} \times e^{-\omega_{m}(\vec{p}) \times_{0}-i \vec{p} \cdot \vec{x}} f(\mathbf{x})
$$

as before, except in this case $f(\mathrm{x})$ is a four-component covariant wave function. Note that

$$
\Lambda_{+}(p)=\frac{\chi(p) \chi^{\dagger}(p)}{2 \omega_{m}(\vec{p})}
$$

is a Hermitian matrix with

$$
\chi(p)=\sqrt{m} \gamma^{0} u_{m}(\overrightarrow{\mathrm{p}})
$$

where $u_{m}(\overrightarrow{\mathrm{p}})$ a Dirac $u$-spinor. The function $\chi(p)$ is a $2 \times 4$ matrix that satisfies the intertwining relation

$$
\Lambda \chi(p)=\chi(\Lambda p) R_{w}(\Lambda, p)
$$


where $R_{w}(\Lambda, p)$ is a Wigner rotation. This matrix intertwines Dirac spinor representations of the Lorentz group with positive-mass positive-energy irreducible representation of the Poincare group.

If we define

$$
\psi(p):=\frac{1}{(2 \pi)^{3 / 2}} \chi^{\dagger}(p) g(\vec{p})
$$

the scalar product

$$
\langle\psi \mid \psi\rangle:=\int \frac{d^{3} p}{2 \omega_{m}(\vec{p})} \psi^{\dagger}(\vec{p}) \psi(\vec{p})
$$

has the standard form of a mass $m$, spin $1 / 2$ irreducible representation of the Poincaré group. Note that two component spinors characteristic of spin $1 / 2$ positive mass irreducible representations of the Poincaré group arise from the factorization (59).

The intertwining relations (61) imply that when the generators (35138) are transformed to act on the Poincaré irreducible wave functions $\psi(p)$ that the generators take on the standard forms

$$
\begin{gathered}
\vec{B}=i \omega_{m} \vec{\nabla}_{\mathrm{p}}+\frac{1}{m+\omega_{m}(p)} \vec{p} \times \vec{j} \\
\vec{J}=i \vec{\nabla}_{\mathrm{p}} \times \overrightarrow{\mathrm{p}}+\vec{j}
\end{gathered}
$$

where $\vec{j}$ is the canonical spin operator that acts on the two-dimensional range of $\chi$

$$
\vec{\Sigma} \chi(p)=\chi(p) \vec{j}_{c}
$$

where $\vec{\Sigma}$ is defined by (41).

This shows how the abstract Euclidean formulation of the relativistic quantum dynamics associated with a set of model Schwinger functions given in section three leads to the standard Minkowski description of the dynamics of a single particle in terms of irreducible representations of the Poincaré group.

Since the many-point Schwinger functions for a system of free particles are made up out of sums of tensor products of two-point functions, this result implies reflection positivity for the full set of free-particle Schwinger functions.

\section{$5 \quad$ Stability}

In this section we investigate the stability of reflection positivity. This is the main result in this paper. Our analysis is limited to the necessary conditions for reflection positivity given by equations (34) and (33).

To gain some insight into the problem we first consider a toy $2 \times 2$ matrix model. We use this model to check stability of reflection positivity in the simplest possible case.

The problem is to consider a model of the inhomogeneous Euclidean BetheSalpeter equation

$$
S=S_{0}+S_{0} K S
$$


where $S_{0}$ is positive and reflection positive. Note that while the positivity of $S$ is not required, for Schwinger functions of scalar fields it is used to derive bounds needed to prove the spectral condition [20. What are the restrictions on $K$ that preserve these properties?

To motivate the matrix model consider the quadratic form $(f, \Theta S f)$ and model $f$ by a constant $f_{c}$ times $\delta(\overrightarrow{\mathrm{x}}) \delta\left(\mathrm{x}^{0}-\mathrm{t}\right)$ with $\mathrm{t}$ positive. We also consider the time reflected point $\mathrm{t}^{\prime}=\theta \mathrm{t}=-\mathrm{t}$. We treat $f$ as a column vector with the upper component corresponding to the value of $f=f_{c}$ at Euclidean time $\mathrm{t}$ and the lower component corresponding to the value $f=0$ at the Euclidean time-reversed point $\theta \mathrm{t}=-\mathrm{t}$.

In this model we define

$$
\Pi_{>}:=\left(\begin{array}{cc}
I & 0 \\
0 & 0
\end{array}\right)
$$

and

$$
\Theta:=\left(\begin{array}{cc}
0 & I \\
I & 0
\end{array}\right)
$$

The quadratic form becomes

$$
(f, \Theta S f)=\left(f_{c}, 0\right)\left(\begin{array}{cc}
0 & I \\
I & 0
\end{array}\right)\left(\begin{array}{cc}
s(\overrightarrow{0}, 0) & s(\overrightarrow{0}, 2 \mathrm{t}) \\
s(\overrightarrow{0},-2 \mathrm{t}) & s(\overrightarrow{0}, 0)
\end{array}\right)\left(\begin{array}{c}
f_{c} \\
0
\end{array}\right)=f_{c}^{2} s(\overrightarrow{0},-2 \mathrm{t})
$$

where $\left(f_{c}, 0\right)$ represents a row vector. In this case reflection positivity on this one-dimensional subspace, analogous to (34), requires that $s(\overrightarrow{0},-2 \mathrm{t})>0$.

We write this in a more abstract form by defining

$$
s_{0}:=\left(\begin{array}{cc}
s_{011} & s_{012} \\
s_{021} & s_{022}
\end{array}\right)=\left(\begin{array}{cc}
s(\overrightarrow{0}, 0) & s(\overrightarrow{0}, 2 \mathrm{t}) \\
s(\overrightarrow{0},-2 \mathrm{t}) & s(\overrightarrow{0}, 0)
\end{array}\right) .
$$

In this notation reflection positivity means

$$
\Pi_{>} \Theta S_{0} \Pi_{>}=\left(\begin{array}{cc}
s_{021} & 0 \\
0 & 0
\end{array}\right) \geq 0
$$

or $s_{021}>0$. Euclidean invariance requires

$$
s_{0}=\Theta s_{0} \Theta
$$

which implies that $s_{0 i j}$ are real and satisfy $s_{011}=s_{022}$ and $s_{012}=s_{021}$. It is straightforward to show the requirements of positivity and reflection positivity in this model are satisfied if

$$
s_{011}>s_{012}>0
$$

which means that the matrix $S_{0}$ must have positive elements with the diagonal ones being larger than the off-diagonal ones. This condition must hold for any $2 \times 2$ sub-matrix associated with times $\pm \mathrm{t}$. For larger matrices it is only a 
necessary condition; however this suggest that in the general case there is a growth condition limiting the size of off diagonal elements relative to diagonal elements.

The next step is to add a perturbation using a kernel $K$. In this case we model the Bethe-Salpeter kernel $K$ by an "Euclidean" invariant $2 \times 2$ Hermitian matrix. If

$$
K=\left(\begin{array}{ll}
k_{11} & k_{21} \\
k_{12} & k_{22}
\end{array}\right)
$$

Hermiticity and Euclidean invariance require that $K$ is real, and $k_{11}=k_{22}$ and $k_{12}=k_{21}$. This means that "kernels" $K$ can be parameterized by vectors in the two-dimensional $\left(k_{11}, k_{12}\right)$ plane. The equation for $S$ is

$$
\left(\begin{array}{ll}
s_{11} & s_{21} \\
s_{12} & s_{22}
\end{array}\right)=\left(\begin{array}{ll}
s_{011} & s_{012} \\
s_{012} & s_{011}
\end{array}\right)+\left(\begin{array}{ll}
s_{011} & s_{012} \\
s_{012} & s_{011}
\end{array}\right)\left(\begin{array}{ll}
k_{11} & k_{12} \\
k_{12} & k_{11}
\end{array}\right)\left(\begin{array}{ll}
s_{11} & s_{21} \\
s_{12} & s_{22}
\end{array}\right) .
$$

This equation can be solved for $S$ and the conditions for positivity and reflection positivity are found to be:

$$
\begin{gathered}
k_{11}+k_{12}<\frac{1}{s_{011}+s_{012}} \\
k_{11}<\frac{s_{011}}{\operatorname{det}\left(S_{0}\right)}=\frac{s_{011}}{s_{011}^{2}-s_{012}^{2}} \\
k_{12}>-\frac{s_{012}}{\operatorname{det}\left(S_{0}\right)}=-\frac{s_{012}}{s_{011}^{2}-s_{012}^{2}} .
\end{gathered}
$$

The important property of these inequalities is that they define a region that contains an open set containing the origin in the $\left(k_{11}, k_{12}\right)$ plane. This means that reflection positivity is preserved in this model for sufficiently small BetheSalpeter kernels. In this trivial model the reflection positivity condition is stable with respect to small variations about the unperturbed system. This simple construction provides clues about the key elements of a general stability construction.

The next step is to consider the actual inhomogeneous Euclidean BetheSalpeter equation. Abstractly we still have the operators $\Pi_{>}, \Theta, S_{0}, S$, and $K$. Euclidean covariance requires

$$
[\Theta, S]=[\Theta, K]=\left[\Theta, S_{0}\right]=0 .
$$

Under the conditions that $S_{0}$ has an inverse the operator $T$ is defined by

$$
T=S_{0}^{-1}+S_{0}^{-1} S S_{0}^{-1}
$$

This can be used to write $S$ in the solved form

$$
S=S_{0}+S_{0} T S_{0}
$$

It follows from (25) and (82) that the Bethe-Salpeter $T$-operator can be determined directly by solving the equation

$$
T=K+K S_{0} T
$$


If the Källén-Lehmann representation of the two-point function has a mass gap and the Euclidean norm of $K$ is sufficiently small this equation has a unique solution.

If $f \in \mathcal{S}_{>}$is a function of two Euclidean space-time variables then the $(\text { norm })^{2}$ of the vector $[f]_{\sim}$ in the interacting theory is given by

$$
\left\|[f]_{\sim}\right\|^{2}=\left(\Theta \Pi_{>} f,\left(S_{0}+S_{0} T S_{0}\right) \Pi_{>} f\right) .
$$

This can be written in the form

$$
\left\|[f]_{\sim}\right\|^{2}=\left(\Pi_{>} f,\left(\Theta S_{0}+S_{0} \Theta T S_{0}\right) \Pi_{>} f\right)
$$

where we have used the Euclidean invariance of $S_{0}$. Equation (34) requires that this form is non-negative if it is interpreted as the norm of a vector in an underlying quantum theory. The main results of the paper are contained in three theorems.

Theorem 1: If $f \neq 0$ satisfies

$$
\left(\Pi_{>} f, \Theta S_{0} \Pi_{>} f\right)=0
$$

then there are arbitrarily small Euclidean covariant Bethe-Salpeter kernels that violate reflection positivity of $S$.

To prove Theorem 1 note that (86) implies that the surviving contribution to equation (84) is

$$
\left\|[f]_{\sim}\right\|^{2}=\left(\Pi_{>} f, S \Pi_{>} f\right)=\left(S_{0} \Pi_{>} f,(\Theta T) S_{0} \Pi_{>} f\right) .
$$

Let

$$
\chi=S_{0} \Pi_{>} f
$$

which gives

$$
\left\|[f]_{\sim}\right\|^{2}=\left(\Pi_{>} f, \Theta S \Pi_{>} f\right)=(\chi, \Theta T \chi) .
$$

Note that $\chi$ is not an element of $\mathcal{S}_{>}$. Since the free $S_{0}$ is invertible on the full Euclidean space, $\chi$ is not zero. An arbitrarily small $\Theta T$ with Euclidean covariant $T$ can be chosen to have non-zero matrix elements on the subspace spanned by the $\chi$ 's. This quantity must be non-negative for this to represent the square of the Hilbert-space norm in the interacting theory.

We are concerned with the stability of the sign of (89) for sufficiently small $T$. If this expression is negative we get a violation of the necessary condition (34) for reflection positivity; if it is positive, then $T \rightarrow-T$ gives a negative result, independent of the size of the perturbation $T$. Note that $T \rightarrow-T$ is equivalent to $K \rightarrow K_{-}$where $K_{-}$is the solution to the integral equation

$$
K_{-}=-K+2 K S_{0} K_{-} .
$$

This will be small if $K$, considered as an operator, has a sufficiently small Euclidean norm. This completes the proof of Theorem 1. 
This is the stability problem that is the key cause for concern in this paper. The reason that the problem did not appear in the $2 \times 2$ matrix model is because the "unperturbed model" was implicitly constructed to have no null space.

The following theorems show that the condition for the instability to occur is satisfied if $S_{0}$ is constructed out of free two-point Schwinger functions. They also show that the exact two-point Schwinger functions do not have this property.

Theorem 2: The null equivalence class of $S_{0},[0]_{\sim}$, contains an infinite number of functions if $S_{0}$ is the Schwinger function of a free field theory:

Theorem 3: The null equivalence class of $S_{0},[0]_{\sim}$, contains no non-zero functions if $S_{0}$ has a Källén-Lehmann weight with an absolutely continuous component of its mass spectrum.

To prove these theorems we note that the general form of the two-point Schwinger function for a scalar field theory is given by its Källén-Lehmann representation

$$
S_{0}\left(\mathrm{x}-\mathrm{x}^{\prime}\right)=\frac{1}{(2 \pi)^{2}} \int d^{4} \mathrm{p} d m \rho(m) \frac{e^{i \mathrm{p}^{0}\left(\mathrm{t}-\mathrm{t}^{\prime}\right)+i \overrightarrow{\mathrm{p}} \cdot\left(\overrightarrow{\mathrm{x}}-\overrightarrow{\mathrm{x}}^{\prime}\right)}}{\mathrm{p}^{2}+m^{2}} .
$$

A function $f(\overrightarrow{\mathrm{x}}, \mathrm{t})$ with positive-time support is in the null equivalence class of $S_{0}$ on the range of $\Pi_{>}$if and only if

$$
\left(f, \Theta S_{0} f\right)=0 .
$$

To see what this means note that

$$
\begin{gathered}
\left(f, \Theta S_{0} f\right)= \\
\int f^{*}(\overrightarrow{\mathrm{x}},-\mathrm{t}) \frac{1}{(2 \pi)^{2}} \int d^{4} \mathrm{p} d m \rho(m) \mathrm{p} \frac{e^{i \mathrm{p}^{0}\left(\mathrm{t}-\mathrm{t}^{\prime}\right)-i \overrightarrow{\mathrm{p}} \cdot\left(\overrightarrow{\mathrm{x}}-\overrightarrow{\mathrm{x}}^{\prime}\right)}}{\left(\mathrm{p}^{0}-i \omega_{m}(\overrightarrow{\mathrm{p}})\right)\left(\mathrm{p}^{0}+i \omega_{m}(\overrightarrow{\mathrm{p}})\right)} f\left(\overrightarrow{\mathrm{x}}^{\prime}, \mathrm{t}^{\prime}\right) d^{4} \mathrm{x} d^{4} \mathrm{x}^{\prime} .
\end{gathered}
$$

Direct calculation of this gives

$$
\left(f, \Theta S_{0} f\right)=\int d^{3} \mathrm{p} d m \rho(m)\left|\int d \mathrm{t} \tilde{f}(\overrightarrow{\mathrm{p}}, \mathrm{t}) \frac{2 \pi e^{-\omega_{m}(\overrightarrow{\mathrm{p}}) \mathrm{t}}}{\sqrt{\omega_{m}(\overrightarrow{\mathrm{p}})}}\right|^{2}
$$

where

$$
\tilde{f}(\overrightarrow{\mathrm{p}}, \mathrm{t}):=\frac{1}{(2 \pi)^{3 / 2}} \int d^{3} x e^{i \overrightarrow{\mathrm{p}} \cdot \vec{x}} f(\overrightarrow{\mathrm{x}}, \mathrm{t}) .
$$

This will vanish if and only if

$$
\int d \mathrm{t} \tilde{f}(\overrightarrow{\mathrm{p}}, \mathrm{t}) \frac{2 \pi e^{-\omega_{m}(\overrightarrow{\mathrm{p}}) \mathrm{t}}}{\sqrt{\omega_{m}(\overrightarrow{\mathrm{p}})}}=0
$$


for all values of $\vec{p}$ and all $m$ in the spectrum of the Källén-Lehmann weight, $\rho(m)$, of the two-point function. A necessary and sufficient condition for $f$ to represent a function in the null equivalence class of $S_{0}$ is

$$
I=\int d \mathrm{t} \tilde{f}(\overrightarrow{\mathrm{p}}, \mathrm{t}) e^{-\omega_{m}(\overrightarrow{\mathrm{p}}) \mathrm{t}}=0
$$

for all $\vec{p}$ and all $m$ in the spectrum of the Källén-Lehmann weight.

We first consider the free field case where there is only a single mass $(\rho(m)=$ $\left.\delta\left(m-m_{0}\right)\right)$. We show how to construct a large class of functions $\tilde{f}(\overrightarrow{\mathrm{p}}, \mathrm{t})$ with support on compact positive Euclidean time intervals $[a, b], 0<a<b<\infty$, in the null equivalence class of $S_{0}$. Let $\chi(\mathrm{t})$ be a Schwartz function with support on $[a, b]$ satisfying the normalization condition

$$
\int_{a}^{b} d \mathrm{t} \chi(\mathrm{t})=1
$$

let $\tilde{g}(\overrightarrow{\mathrm{p}}) e^{b \omega_{m}(\mathrm{p})}$ be a Schwartz function of $\overrightarrow{\mathrm{p}}$ and let

$$
h(\overrightarrow{\mathrm{p}}):=\int d \mathrm{t} e^{-\omega_{m}(\overrightarrow{\mathrm{p}}) \mathrm{t}} \chi(\mathrm{t}) .
$$

Define

$$
\tilde{f}(\overrightarrow{\mathrm{p}}, \mathrm{t}):=\chi(\mathrm{t}) \tilde{g}(\overrightarrow{\mathrm{p}}) \times\left[1-e^{\omega_{m}(\mathrm{p}) t} h(\overrightarrow{\mathrm{p}})\right] .
$$

By construction $\tilde{f}(\overrightarrow{\mathrm{p}}, \mathrm{t})$ is a non-trivial function with compact positive-time support on $[a, b]$ satisfying $\left(f, \Theta S_{0} f\right)=0$.

In the Euclidean Bethe-Salpeter equation $S_{0}$ is a sum of products of twopoint Schwinger functions. From the discussion above, if the individual Schwinger functions have single masses then it is possible to find functions with support in any compact positive time interval that are in the null equivalence class of $S_{0}$. Choosing products of functions with disjoint positive time support it is possible to find functions in the range of $\Pi_{>}$that are in the null equivalence class of the tensor product of two $\Theta S_{0}$ 's.

On the other hand it is clear that $\Theta S_{0} f$ is non-zero since for $g=\Theta f$

$$
\begin{aligned}
\left(g, \Theta S_{0} f\right)=\left(f, S_{0} f\right)= & \frac{1}{(2 \pi)^{4}} \int d^{4} \mathrm{p} d^{4} \times d^{4} \mathrm{y} f^{*}\left(\overrightarrow{\mathrm{x}}, \mathrm{t}_{x}\right) \frac{e^{i \mathrm{p} \cdot(\mathrm{x}-\mathrm{y})}}{\mathrm{p}^{2}+m^{2}} f\left(\overrightarrow{\mathrm{y}}, \mathrm{t}_{y}\right)= \\
& \int d^{4} \mathrm{p} \frac{\left|\hat{f}\left(\overrightarrow{\mathrm{p}}, \mathrm{p}^{0}\right)\right|^{2}}{\mathrm{p}^{2}+m^{2}}>0,
\end{aligned}
$$

where $\hat{f}\left(\overrightarrow{\mathrm{p}}, \mathrm{p}^{0}\right)$ is the four dimensional Fourier transform of $f\left(\overrightarrow{\mathrm{x}}, \mathrm{t}_{x}\right)$. This implies that for any $f$ in the null equivalence class of $S_{0}$ there are functions $g$ with no support restrictions that satisfy

$$
\left(g, \Theta S_{0} f\right) \neq 0 .
$$

If there are exchange contributions to the Green functions, we have

$$
\left(\left[f_{1}\right]_{\sim}\left[f_{2}\right]_{\sim}, \Theta S_{0}\left[f_{1}\right]_{\sim}\left[f_{2}\right]_{\sim}\right)=
$$




$$
\left(\left[f_{1}\right]_{\sim}, \Theta S_{01}\left[f_{1}\right]_{\sim}\right)\left(\left[f_{2}\right]_{\sim}, \Theta S_{01}\left[f_{2}\right]_{\sim}\right)+\left(\left[f_{1}\right]_{\sim}, \Theta S_{01}\left[f_{2}\right]_{\sim}\right)\left(\left[f_{2}\right]_{\sim}, \Theta S_{01}\left[f_{1}\right]_{\sim}\right)
$$

which is zero if $\left[f_{1}\right]_{\sim}=\left[f_{2}\right]_{\sim}=[0]_{\sim}$.

It follows that if $S_{0}$ is the $S_{0}$ of a free field that $\Pi_{>} \Theta S_{0} \Pi_{>}$has a non-trivial null space on the range of $\Pi_{>}$. This completes the proof of Theorem 2.

The analysis above also applies to the spin $1 / 2$ case because the $t$ dependence in (97) and (58) is identical.

This shows that solutions of the Euclidean Bethe-Salpeter equation formulated with a free $S_{0}$ are not reflection-positivity stable with respect to small perturbations. This means one can alway find arbitrarily small Bethe-Salpeter kernels that make $\left\|[f]_{\sim}\right\|^{2}=\left([f]_{\sim}, \Theta S[f]_{\sim}\right)<0$, which violates reflection positivity.

Since the two-point functions that appear in the exact Bethe-Salpeter equation of a local field theory are not generally sums of products of free Schwinger functions, it is worth investigating if these more realistic two-point functions have a non-trivial null equivalences classes. Theorem 3 addresses this question.

To prove Theorem 3 fix $\vec{p}$. The condition for $\tilde{f}(\overrightarrow{\mathrm{p}}, \mathrm{t})$ to be in the null equivalence class of $S_{0}$ is (97).

If $\tilde{f}(\overrightarrow{\mathrm{p}}, \mathrm{t})$ is a Schwartz function in $\mathrm{t}$ with support for positive $\mathrm{t}$ then for fixed $\vec{p}$

$$
F(z):=\int d \mathrm{t} \tilde{f}(\overrightarrow{\mathrm{p}}, \mathrm{t}) e^{-z \mathrm{t}}
$$

is an analytic function for $\Re(z)>0$. As $m$ varies continuously in the spectrum of the Källén-Lehmann weight $z=\omega_{m}(\vec{p})=z(m)$ traces out a real interval in the domain of analyticity of $F(z)$, where $F(z(m))=0$. Since $F(z)$ is analytic in the right half plane, it must be identically zero on the entire domain of analyticity. If $\tilde{f}(\overrightarrow{\mathrm{p}}, \mathrm{t})$ is a Schwartz function in $\mathrm{t}$ for fixed $\overrightarrow{\mathrm{p}}$ this is continuous on the boundary as $y \rightarrow 0$. It follows that

$$
\int d \mathrm{t} \tilde{f}(\overrightarrow{\mathbf{p}}, \mathrm{t}) e^{-i y t}=\lim _{x \rightarrow 0^{+}} F(x+i y)=0
$$

which means that the Fourier transform of $\tilde{f}(\overrightarrow{\mathrm{p}}, \mathrm{t})$ in $\mathrm{t}$ is identically zero. This same argument can be applied to each $\vec{p}$. This proof can be extended to the case that $\tilde{f}(\overrightarrow{\mathrm{p}}, \mathrm{t})$ is a tempered distribution in $\mathrm{t}[21]$.

Since this can be done for any $\vec{p}$ it follows that there are no non-zero functions in the null equivalence class of $S_{0}$ if the spectrum of Källén-Lehmann weight has any absolutely continuous component. This completes the proof of Theorem 3.

This is an encouraging result that leaves open the possibility that a stability result might be possible if the Bethe-Salpeter equation is formulated with a more realistic two-point function in the driving term. 


\section{Conclusion}

In this paper we proved that the solution of the Euclidean Bethe-Salpeter equation

$$
S=S_{0}+S_{0} K S
$$

with $S_{0}$ a free field Schwinger function can violate reflection positivity for arbitrarily small Euclidean covariant kernels $K$. When reflection positivity is violated, the standard axiomatic construction of the physical Hilbert space leads to a pathological inner product with negative (norm) ${ }^{2}$ vectors, making it impossible to give the theory a quantum mechanical interpretation.

The basis of the instability is simple to understand. In quantum field theory the quantum mechanical scalar product can be expressed directly in terms of the Schwinger functions as

$$
\langle f \mid g\rangle:=\left(\Pi_{>} f, \Theta S \Pi_{>} g\right) .
$$

The Schwinger function can be expressed using a cluster expansion as the sum of a linked term and unlinked term

$$
S=S_{0}+S_{0} T S_{0}
$$

where $T$ is Euclidean covariant. The Euclidean Bethe-Salpeter equation generates the linked terms in terms of the Bethe-Salpeter kernel, $K$. If a test function $f$ in the range of $\Pi_{>}$satisfies $\left(\Pi_{>} f, \Theta S_{0} \Pi_{>} f\right)=0$ then the expression for the square of the norm of the corresponding vector is

$$
\|f\|^{2}:=\left(\Pi_{>} f, S_{0} \Theta T S_{0} \Pi_{>} f\right)=\left(S_{0} \Pi_{>} f, \Theta T S_{0} \Pi_{>} f\right) .
$$

If we pick an arbitrary Euclidean covariant $T$ satisfying $\|T\|_{e}<\epsilon$ that makes the above expression non-zero, then either the (norm) $)^{2}$ is negative or it can be made negative by changing the sign of $T$. In either case we end up with an instability for an arbitrarily small perturbation $T$. Similar remarks apply to the kernel $K$.

The existence of the instability depends on the existence of functions of Euclidean space-time variables with Euclidean-time support in the range of $\Pi_{>}$ that satisfy $\left(f, \Theta S_{0} f\right)=0$. We exhibited a large class of these functions for the case that $S_{0}$ is the $S_{0}$ of a free field theory. Conversely, we argued that there are no such functions when the Källén-Lehmann weight of the two-point function includes a continuous mass spectrum. This suggests that the instability only impacts model Bethe-Salpeter equations where the driving term is replaced by the free $S_{0}$

The problems with the sign of the Hilbert space norm are unrelated to sign problems that sometimes occur with the normalization of Bethe-Salpeter amplitudes 22. The signs associated with the Bethe-Salpeter normalization condition

are directly related to the normalization of the Green function, however they say nothing about the underlying Hilbert space of the theory. 
Negative norms can appear in gauge theories. In the case of gauge theories negative norms arise because the space generated by applying polynomials of the field operators to the vacuum includes unphysical degrees of freedom. The problem identified in this paper occurs in models of theories where polynomials in the fields applied to the vacuum only generate physical states. Thus the negative norms identified in this paper are associated with vectors that should represent physical states. An investigation of the analogous stability question in a gauge theory is beyond the scope of this paper.

Another question is whether the constraint of Euclidean covariance is the appropriate minimal constraint on the model Bethe-Salpeter kernels. We were unable to identify other generic constraints which are motivated by the structure of local quantum field that would prevent the instability.

Our interest in this instability arose from attempts to construct a robust class of relativistic quantum models based on solutions of a Euclidean Bethe-Salpeter equation with the driving term being the $S_{0}$ of a free field theory. One goal was to identify a class of model Euclidean-Bethe Salpeter kernels, for example small, Euclidean-covariant separable kernels, that could be used to construct relativistic quantum models, under the assumption that the resulting model model-Schwinger functions have the same relation to the underlying quantum theory as the exact Schwinger functions. The identification of the instability in this paper shows that this problem has no solution if the kernels are allowed to be arbitrary Euclidean covariant kernels with sufficiently small Euclidean norms.

This work suggests that an interesting problem is to investigate the more realistic case, where the weight of the Källén-Lehmann representation of the twopoint function has a non-empty absolutely continuous spectrum. The absence of a null space suggest that it might be possible to generalize the analysis of the matrix model to to find bounds on the kernel, expressed in terms of $S_{0}$, that might lead to stability. A result of this type would provide useful restrictions on model Bethe-Salpeter kernels that ensure a relativistic quantum interpretation.

The authors have benefited from useful discussions with P.E.T. Jorgensen, F. Coester, M. Fuda and D. Phillips. 


\section{References}

[1] E. P. Wigner: Ann, Math, 40,149(1939)

[2] I. Montvay and G. Münster: Quantum Fields on a Lattice Cambridge Monographs on Mathematical Physics, 1997, p 8-15

[3] P. Maris and C. D. Roberts: Int.J.Mod.Phys. E12:297-365(2003)

[4] K. Osterwalder and R. Schrader: Communications in Mathematical Physics 31,83(1973)

[5] J. Fröhlich: Helvetica Physics Acta, 47,265(1974)

[6] K. Osterwalder, R. Schrader: Communication in Mathematical Physics $\mathbf{4 2 , 2 8 1 ( 1 9 7 5 )}$

[7] I. M. Gelfand and G. E. Shilov: Generalized Functions, Volume 2, Academic Press, 1968

[8] B. Simon: J. Math. Phys. 12,140(1971), see Theorem 1

[9] W. N. Polyzou: Phys. Rev. D32,995(1985)

[10] F. J. Dyson: Phys. Rev. 75,1736(1949).

[11] J. Schwinger: Proceedings of the National Academy of Sciences $\mathbf{4 4 , 9 5 6}$ (1958).

[12] R. F. Streater and A. S. Wightman: PCT, Spin and Statistics, and All That, Princeton Landmarks in Physics, 1980, sec. 2.3

[13] R. F. Streater and A. S. Wightman: PCT, Spin and Statistics, and All That, Princeton Landmarks in Physics, 1980

[14] E. Nelson: J. Funct. Analysis 12,97(1973)

[15] J. Fröhlich: Advances in Mathematics 23,119(1977)

[16] A. Klein, L. Landau: Journal of Functional Analysis 44,121(1981)

[17] A. Klein, L. Landau: Communication in Mathematical Physics $\mathbf{8 7 , 4 6 9 ( 1 9 8 3 )}$

[18] Fröhlich, J, Osterwalder, K., Seiler, E.: Annals of Mathematics, 118,461(1983)

[19] F. Riesz and B. Sz-Nagy: Functional Analysis, Dover, 1990, see p. 395

[20] J. Glimm and A. Jaffe: Quantum Physics, Springer Verlag, 1996

[21] M. Reed and B. Simon:Methods of Modern Mathematical Physics, Volume II, Academic Press, 1975, Theorem XI.16 
[22] N. Nakanishi: Prog. Theor. Phys. Suppl. 95,1(1988)

\section{Appendix: The Complex Euclidean/Lorentz Group}

The complex Lorentz group 13 and complex $O(4)$ are the same group. The covering group of both groups is $S L(2, C) \times S L(2, C)$. To illustrate this connection let $x$ be a real Lorentz four vector. Let

$$
\sigma_{\mu}=\left(I, \sigma_{1}, \sigma_{2}, \sigma_{3}\right)
$$

be the $2 \times 2$ identity matrix and the three $2 \times 2$ Pauli spin matrices. Define the Hermitian $2 \times 2$ matrix

$$
X:=x^{\mu} \sigma_{\mu}=\left(\begin{array}{cc}
x^{0}+x^{3} & x^{1}-i x^{2} \\
x^{1}+i x^{2} & x^{0}-x^{3}
\end{array}\right) .
$$

This definition implies:

$$
x^{\mu}=\frac{1}{2} \operatorname{Tr}\left[\sigma_{\mu}^{\dagger} X\right]
$$

The determinant of the matrix $X$,

$$
\operatorname{det}(X)=-x \cdot x=\left(x^{0}\right)^{2}-\vec{x} \cdot \vec{x},
$$

is the Lorentz invariant length of $x$. Also note that for real $x, X^{\dagger}=X$.

The set of non-trivial linear transformations that preserve both $\operatorname{det}(X)$ and $X=X^{\dagger}$ have the form:

$$
X^{\prime}=\Lambda X \Lambda^{\dagger}
$$

where $\Lambda$ is any complex $2 \times 2$ matrix with $\operatorname{det}(\Lambda)=1$. These matrices have a 2 to 1 correspondence with the real Lorentz transformations continuously connected to the identity:

$$
\Lambda_{\nu}^{\mu}:=\frac{1}{2} \operatorname{Tr}\left[\sigma_{\mu} \Lambda \sigma_{\nu} \Lambda^{\dagger}\right]
$$

with $\Lambda$ and $-\Lambda$ corresponding to the same real Lorentz transformation. This can be derived by multiplying (114) by $\sigma_{\mu}$ and taking the trace using the trace formula (112). Rotations correspond to the case that $\Lambda$ is unitary while rotationless boosts correspond to the case the $\Lambda$ is a positive matrix.

Including indices on $X \rightarrow X_{a b}$ the transformation properties are

$$
X_{a b}^{\prime}=\Lambda_{a}^{a^{\prime}} \otimes\left(\Lambda^{*}\right)_{b}^{b^{\prime}} X_{a^{\prime} b^{\prime}} .
$$

This shows that a four vector transforms covariantly with respect to $\Lambda \otimes$ $\Lambda^{*}$. For a general complex $\Lambda, \Lambda$ and $\Lambda^{*}$ define inequivalent representations of $S L(2, C)$; they cannot be related by a similarity transformation. These are the fundamental representation of $S L(2, C)$ and are the building blocks of all (finite dimensional) spinor and tensor representations of the Lorentz group. 
In a similar manner let $\mathrm{x}$ be a real Euclidean four vector. Let

$$
\sigma_{e \mu}=\left(i I, \sigma_{1}, \sigma_{2}, \sigma_{3}\right)
$$

and

$$
\mathrm{X}:=\mathrm{x}^{\mu} \sigma_{e \mu}=\left(\begin{array}{cc}
i \mathrm{x}^{0}+\mathrm{x}^{3} & \mathrm{x}^{1}-i \mathrm{x}^{2} \\
\mathrm{x}^{1}+i \mathrm{x}^{2} & i \mathrm{x}^{0}-\mathrm{x}^{3}
\end{array}\right)
$$

This can be inverted using multiplication by the Pauli matrices and taking traces:

$$
\mathrm{x}^{\mu}=\frac{1}{2} \operatorname{Tr}\left(\sigma_{e \mu}^{\dagger} \mathrm{X}\right) .
$$

Note that

$$
\operatorname{det}(X)=-x \cdot x=-\left(x^{0}\right)^{2}-\vec{x} \cdot \vec{x}
$$

which is $(-)$ the Euclidean invariant (length) $)^{2}$ of $x$. Also note for real $x$ that $\mathrm{X}=-\sigma_{2} \mathrm{X}^{*} \sigma_{2}$.

This condition is preserved for

$$
\mathrm{X}^{\prime}=A \mathrm{X} B^{t}
$$

provided both $A$ and $B$ are unitary. In terms of the individual Euclidean components

$$
E^{\mu}{ }_{\nu}=E(A, B)^{\mu}{ }_{\nu}:=\frac{1}{2} \operatorname{Tr}\left[\sigma_{e \mu}^{\dagger} A \sigma_{e \nu} B^{t}\right] .
$$

This can be derived by multiplying (121) by $\sigma_{e \mu}^{\dagger}$ and taking the trace using the trace formula (119).

Including indices on the $\mathrm{X} \rightarrow \mathrm{X}_{a b}$ then the transformation properties are

$$
\mathrm{X}_{a b} \rightarrow \mathrm{X}_{a b}^{\prime}=A_{a}{ }^{a^{\prime}} \otimes B_{b}{ }^{b^{\prime}} \mathrm{X}_{a^{\prime} b^{\prime}} .
$$

This shows that a Euclidean four vector transforms covariantly with respect to $A \times B$.

The restrictions $X^{\prime}=A X A^{\dagger}$ with $\operatorname{det}(A)=1$ and $\mathrm{X}^{\prime}=A \mathrm{X} B^{t}$ with $A$ and $B$ unitary are designed to keep the components of the Minkowski or Euclidean four vectors real. For $A$ and $B$ arbitrary complex $2 \times 2$ matrices with $\operatorname{det}(A)=$ $\operatorname{det}(B)=1$ the invariant length (Minkowski or Euclidean) is still preserved. What changes is that the individual components of the vectors are complex. The complex transformations that act on Lorentz or Euclidean four vectors are

$$
\Lambda(A, B)^{\mu}{ }_{\nu}:=\frac{1}{2} \operatorname{Tr}\left[\sigma_{\mu} A \sigma_{\nu} B^{t}\right]
$$

and

$$
E(A, B)^{\mu}{ }_{\nu}:=\frac{1}{2} \operatorname{Tr}\left[\sigma_{e \mu}^{\dagger} A \sigma_{e \nu} B^{t}\right] .
$$

These two representation differ by a similarity transformation that multiplies Minkowski time by $i$ to get the Euclidean time. This means the any four vector has Euclidean and Minkowski components, $\mathrm{x}^{\mu}$ and $x^{\mu}$ respectively. Under $S L(2, C) \times S L(2, C)$ they transform like

$$
x^{\mu} \rightarrow x^{\prime \mu}=\Lambda(A, B)^{\mu}{ }_{\nu} x^{\nu}
$$


or equivalently

$$
\mathrm{x}^{\mu} \rightarrow \mathrm{x}^{\prime \mu}=E(A, B)^{\mu}{ }_{\nu} \mathrm{x}^{\nu} .
$$

The Lie algebra of the Lorentz group can be derived by considering the appropriate infinitesimal complex Euclidean transformations

Transformation properties of Euclidean Green functions can be determined by the transformation properties of the corresponding Minkowski functions by replacing the fundamental representations $\left(\Lambda, \Lambda^{*}\right)$ by the pair of $S U(2)$ matrices $(A, B)$.

In applications it is important to understand the transformation properties of tensor and spinor quantities with respect to Euclidean transformations, given a knowledge of the transformation properties of the corresponding Minkowski quantities. In general the transition is made from the finite dimensional representation $D\left(\Lambda, \Lambda^{*}\right)$ of the Lorentz group to the finite dimensional representation $D(A, B)$ of the four dimensional orthogonal group by making the replacements $\Lambda \rightarrow A$ and $\Lambda^{*} \rightarrow B$. 